Med Klin Intensivmed Notfmed 2013 .

108:78-78

DOI 10.1007/s00063-013-0216-4

c) Springer-Verlag Berlin Heidelberg 2013

\section{M.T. Geier}

Klinik und Poliklinik für Physikalische Medizin und

Rehabilitation, Klinikum der Universität München

\title{
Was ist Physiotherapie in der Intensivmedizin?
}

Es gibt nicht die Physiotherapie auf Intensivstationen, sondern die Physiotherapie beinhaltet eine Vielzahl von Methoden, Konzepten und Techniken, die am Patienten angewendet werden.

Die Physiotherapie auf Intensivstationen arbeitet nach folgenden Prinzipien: Struktur erhalten, Funktion fördern und dadurch Förderung der Aktivität und Partizipation (ICF-Modell).

Durch physiotherapeutische Interventionen setzen wir Reize, die direkt und/oder indirekt die Wahrnehmung/ das Bewusstsein, die Atmung, das HerzKreislauf-System, den Stoffwechsel und die Motorik des Patienten beeinflussen.

Für die Atmung würde das z. B. bedeuten, dass wir beim kontrolliert beatmeten Patienten strukturerhaltend (Erhalt der muskuloskelettalen Anteile der Atempumpe) arbeiten. Bei assistiert beatmeten bzw. spontan atmenden $\mathrm{Pa}$ tienten liegt der therapeutische Schwerpunkt im Erhalten und Fördern der Funktion (Optimierung der physiologischen Atmung).

Grundlage für jede physiotherapeutische Behandlung ist die fachspezifische Befunderhebung.

Die angewandten Methoden beruhen auf den 3 Grundsäulen der Physiotherapie: manuelle Techniken, Bewegungstherapie und physikalische Therapie.

Durch die Vielzahl von Methoden und Techniken, die zudem sehr individuell auf den Patienten angepasst werden, ist es sehr schwierig, einen evidenzbasierten Nachweis für die Wirksamkeit unserer Behandlungsmethoden zu erbringen.

Unsere Erfahrung in der Physiotherapie auf Intensivstationen zeigt, dass eine optimal durchgeführte physiotherapeu- tische Behandlung (in guter Zusammenarbeit im interdisziplinären Team) hilft, Sekundärschäden zu vermeiden und das Rehabilitationspotenzial des Patienten zu erhalten und zu fördern.

In weiteren Beiträgen will die Physiotherapie ihre therapeutischen Vorgehensweisen vorstellen:

- An welchen Parametern orientiert sich die Physiotherapie? (Welche Informationen braucht der/die PhysiotherapeutIn für seine effektive Arbeit?),

- fachspezifischer Befund,

- Wahrnehmung/Bewusstsein,

- Atemtherapie (abhängig von der Beatmungsform),

- Herz-Kreislauf-System,

- Stoffwechsel,

- Motorik,

- Wie werden Strukturen erhalten, Funktionen erhalten und gefördert bzw. gefordert?

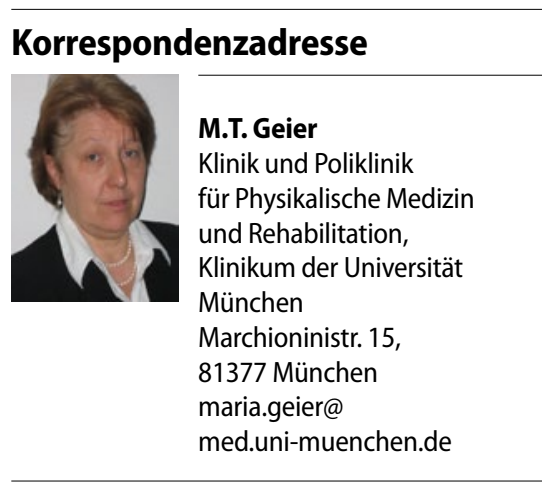

Dieser Beitrag erschien zuerst online-first in der Intensivmedizin und Notfallmedizin DOI 10.1007/ s00390-009-0126-0. 